\title{
Importance of breast milk in the development of intestinal microbiota
}

\author{
Ana M. Castro ${ }^{1 *}$, Marcela V. González ${ }^{2}$, Fernando Constantino ${ }^{2}$ and Fernando M. Galindo \\ ${ }^{1}$ Departamento de Salud Pública, Facultad de Medicina; ${ }^{2}$ Facultad de Medicina Veterinaria y Zootecnia. National Autonomous University of Mexico, \\ Mexico City, Mexico
}

\begin{abstract}
At birth, mammals make the transition from aseptic surroundings to a pathogen-filled environment, it is known that different factors modulate immune responses, but in the digestive tract breast milk (BM) may play an important role in the maturation of the immune response, mainly in relation to the gut. In this sense, it has been demonstrated that besides nutrients and antibodies, BM contains cells, hormones, cytokines, and growth factors. The objective of this work was to evaluate the importance of BM in the development of both the piglet and the intestinal microbiota. For this, two groups of piglets were formed; one suckled with the saw and the other suckled with BM substitute. Stool samples were taken for bacterial identification, this was done through the VITEK-system, and subsequently, the serotype was identified. The results showed that the piglets suckled with BM substitute $18(100 \%)$ presented diarrheal episodes from the $2^{\text {nd }}$ day, of this group two piglets presented bloody diarrhea, and the group fed with the mother only 7 (38.8\%) had diarrheic episodes from the $5^{\text {th }}$ day. All isolates 285 were Escherichia coli. The largest number of isolates obtained of piglets suckled with BM belonged to serotype 0103:H28 and largest number of isolates obtained of piglets suckled with milk substitute belonged to serotype 0166:H21. Serotypes belonged to bloody diarrhea were 0103:H28 and 0166:H21. The present work showed the importance of BM both in the body development of the piglet and in the protection against infections.
\end{abstract}

Key words: Piglets suckled with the saw. Piglets suckled with breast milk substitute. Escherichia coli. Serotypes.

\section{Introduction}

At birth, piglets are devoid of gamma globulins, because the epitelio chondrial placenta of the sows, does not allow the passage of immunoglobulins to the fetus, and passive immunity can be acquired through colostral milk during the first $36 \mathrm{~h}$ of life ${ }^{1}$. To the birth, the gastrointestinal tract of the pig is sterile, but within a few hours, it is colonized by microorganisms. The colonizing bacteria are proposed to be mainly acquired from maternal feces, skin, and teats. Escherichia coli cells, together with Streptococci of Lancefield groups D and $\mathrm{K}$ and Clostridium perfringens, are among the earliest bacteria to colonize the gut in piglets ${ }^{2}$. The composition of the intestinal $E$. coli microbiota in pigs is very complex; these bacteria are considered normal inhabitants of the intestinal tract, but specific serotypes being an important cause of diarrhe ${ }^{2}$. It is known that breastfeeding protects against acute infections of the digestive and respiratory tracts ${ }^{3,4}$ and that intestinal flora is modified by feeding.

At birth, mammals make the transition from aseptic surroundings to a pathogen-filled environment. The mucosal epithelial layer forms the interface between the

\section{Correspondence:}

${ }^{\star}$ Ana M. Castro

E-mail: amcastro@unam.mx
Available online: 30-07-2019

Date of reception: 28-02-2018 DOI: 10.24875/HGMX.M19000023
Rev Med Hosp Gen Mex. 2019;82(2):61-66 www.hospitalgeneral.mx

0185-1063/@ 2018 Sociedad Médica del Hospital General de Mexico. Published by Permanyer México SA de CV. This is an open access article under the CC BY-NC-ND license (http://creativecommons.org/licenses/by-nc-nd/4.0/). 
external and internal environments of the gastrointestinal tract (GIT). This area is the site for digestion and absorption of various essential nutrients, yet it must also function as a barrier against various harmful agents and infectious pathogens ${ }^{2-4}$. It is known that different factors modulate immune responses, but in the digestive tract breast milk (BM) may play an important role in the maturation of the immune response, mainly in relation to the gut. In this sense, it has been demonstrated that besides nutrients and antibodies, BM contains cells ${ }^{5,6}$, hormones, cytokines, and growth factors ${ }^{7,8}$ as a proline-rich protein $^{9}$ and an epidermal growth factor $(E G F)^{10}$. All of these biologically active factors not only facilitate the development of essential digestive functions but also regulate the maturation of the intestinal mucosal barrier. A colostral proline-rich protein induces the growth and differentiation of resting B-cells. The capacity of this protein to induce both B-cell growth and differentiation may provide evidence that its central role is the establishment of an immune response in neonates ${ }^{9}$. EGF is a peptide that produces a variety of biological responses, most of which involve regulation of cell replication, cell movement, and cell survival. In the GIT, EGF not only enhances proliferation and differentiation of epithelial cells but also has significant effects on healing damaged mucosa or on intestinal adaptation after injury ${ }^{10}$. The amino acid, L-glutamine is also present in BM and plays a dual metabolic role in new-born pig enterocytes acting as both a primary metabolic fuel and as an essential source of carbon and nitrogen for biosynthetic processes ${ }^{11}$. In addition, L-glutamine is important for lymphocyte proliferation and generation of lymphokines ${ }^{12}$.

The etiology, epidemiology, and pathogenesis of E. coli causing pre- and post-weaning diarrhea in pigs have been the subject of many studies. However, there is relatively little information about the gut microbiota and their relationship with $E$. coli in piglets suckled with $B M$ and piglets suckled with a milk substitute.

E. coli is a Gram-negative microorganism belonging to the Enterobacteriaceae family, which is usually found, as commensal, in the enteric tract of the human and of many animal species. However, several E. coli strains can cause a wide range of clinical symptoms in humans and animals, due to the expression of particular virulence factors, whereby $E$. coli strains have been classified in different pathogroups ${ }^{13}$.

E. coli is a common porcine enteric pathogen, causing diarrhea in newborn and weaned pigs and edema disease in piglets after weaning. Enterotoxigenic $E$. coli (ETEC) and Shiga toxin-producing E. coli (STEC) are the main categories of diarrheagenic $E$. coli that cause enteric infections in pigs ${ }^{4}$.

ETEC is an E. coli that produces at least one member of two defined groups of enterotoxins, which are able to exert their action on the intestinal epithelium. They are heat-labile (LT) and heat-stable enterotoxins (STs). STs are classified as STa (also called STI) and STb (called (STII) ${ }^{13}$. Most ETEC isolates from diarrheic pigs can produce one or more of the following fimbriae: F4 (K88), F5 (K99), F6 (987P), F17, F18, and F41 14-16. Fimbriae are surface proteins that are responsible for adhesion to intestinal epithelial cells ${ }^{15,16}$. The fimbrial adhesins allow the union of the bacteria to the recipients of the microvilli of the enterocytes; this is followed by the secretion of toxins that are responsible for the production of diarrhea. Once E. coli has colonized the intestine, diarrhea occurs, this may be watery or inflammatory, watery diarrhea is due to the production of one or more enterotoxins and inflammatory diarrhea to the production of cytotoxins ${ }^{13}$.

Diarrhea associated to ETEC belong to a limited number of serogroups of $E$. coli, O8, O9, 020, 064, 0101, 0138, 0141, 0147, O149, and 0157 being the most commonly found in several countries ${ }^{16,17}$.

The second pathotype of $E$. coli is Shiga toxin-producing (STEC) also called verotoxin-producing E. coli, it causes inflammatory diarrhea. E. coli O157:H7 produce the toxin STx which is absorbed from the intestine and enters the bloodstream where it causes systemic damage, resulting in edema disease. However, in addition to E. coli O157:H7, other kinds of STEC, called "non-O157 STEC," cause human diseases. The Centers for Disease Control and Prevention estimate that non-O157 STEC are responsible for about 1579 confirmed cases of illness annually ${ }^{18}$, but cause relatively fewer cases of the hemolytic uremic syndrome (HUS) than E. coli O157:H7. Of these serotypes, serotype 0103 was the first non-O157 STEC strain identified as a suspected cause of sporadic cases of HUS, in 1975 in France.

\section{Materials and methods}

\section{Feeding protocol}

Piglets of the race (hybrids of York X Landrace) they were randomly distributed into two groups; the first one suckled with the saw formed by 18 pigs and the second was suckled with BM substitute formed by 18 pigs.

Milk substitute Lacto Spray Pig (Humelco, The Netherlands) contains all the nutrients in the same 
concentrations that the milk of the sow. Piglets were suckled the first 2 weeks each hour at libitum $(30-40 \mathrm{ml}$ approximately) of Lacto Spray Pig, during $24 \mathrm{~h}$, the $3^{\text {rd }}$ and $4^{\text {th }}$ weeks each hour at libitum $(60-70 \mathrm{ml}$ approximately). As much the water where upon prepared milk as the baby's bottles for each taking, were previously sterilized. As of the $2^{\text {nd }}$ day of life, was added to the feeding Pedialyte (ABBOT) at libitum. Unique dose, $0.5 \mathrm{ml}$ of oxytetracycline (Pfeizer, México) intramuscular shot was applied to all the piglets (with or without diarrheic evacuations) at the $3^{\text {rd }}$ and $5^{\text {th }}$ days of life.

\section{E. coli isolates and their characterization}

Samples were obtained from two piglets group from rectal swabs in piglets suckled with BM and suckled with BM substitute. Samples of feces were obtained of piglets with diarrhea and without diarrhea.

A total of 285 samples were isolated. The collected specimens from each pig were transported in Cary and Blair medium (DIBICO, S.A. de C.V, México), stored to $4^{\circ} \mathrm{C}$ and spread in MacConkey agar, Salmonella Shigella agar, Lactose Sacarosa Xilosa agar, and Green Brilliant agar. Plates were incubated at $37^{\circ} \mathrm{C}$ aerobically overnight. Five lactose-fermenting colonies with the appearance of $E$. coli were randomly selected of each agar milieu and cultured on sheep blood agar plates. Plates were incubated aerobically overnight. E. coli isolates were stored on Tryptic soy agar, and biochemical characteristics were established by VITEK-system (Bio Mérieux, Vitek, Inc. Missouri USA). Then, the isolates were serotypified and kept on Dorset Milieu at RT.

\section{Serotyping}

The determination of $\mathrm{O}$ and $\mathrm{H}$ antigens was carried out in the E. coli Reference Laboratory, Departamento de Salud Pública, Facultad de medicina UNAM, employing all available $\mathrm{O}(\mathrm{O} 1-\mathrm{O} 181)$ and $\mathrm{H}(\mathrm{H} 1-\mathrm{H} 56)$ antisera. Antisera were obtained and absorbed with the corresponding cross-reacting antigens to remove the non-specific agglutinins.

\section{Statistical analysis}

The square Chi-square test was used to compare diarrhea episodes of in both piglets groups, feeding with BM and feeding with milk substitute; as well as the association between the type of lactation and the presence of $E$. coli virulence genes. The statistical analysis was examined by SPSS for Windows 20.0.

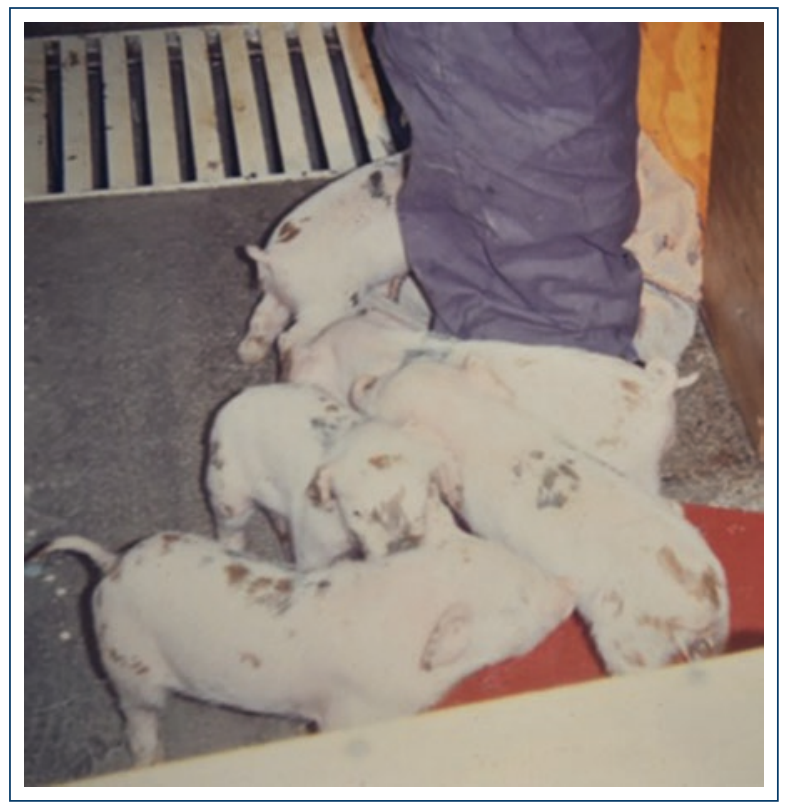

Figure 1. Photo that shows the piglets fed with a breast milk substitute. A student is standing and she is feeding a piglet and other piglets surround her waiting for her food.

\section{Results}

The results showed that the piglets suckled with BM substitute (Fig. 1), 18 (100\%) presented diarrheal episodes from the $2^{\text {nd }}$ day, of this group two piglets presented bloody diarrhea on day 10 of age; and the group fed with the mother (Fig. 2), only 7 (38.8\%) had diarrheic episodes from the $5^{\text {th }}$ day. VITEK system showed that all 285 isolated were $E$. coli.

The $95 \%$ confidence interval was $0.24-0.62$, which suggests that feeding with maternal milk represents a protective factor for the presence of diarrhea in piglets; the fact that they were fed by the mother reduced the risk of diarrhea by $61 \%$. The number needed to treat value of 1.6 indicates that all the piglets that received maternal milk, avoided early diarrhea.

Isolates 285, 164 (57.5\%) come from piglets suckled with BM, which $69.5 \%$ were obtained of piglets healthy and $30.5 \%$ of piglets with diarrhea. Isolates obtained from piglets suckled with milk substitute 121 (42.5\%) were obtained of piglets with diarrhea, which $10 \%$ isolates belonged to bloody diarrhea. The statistical analysis shows significant differences in diarrheic episodes between piglets fed with the mother and piglets fed with maternal milk substitute $(p=0.000)$.

It is important to note that at 1 month of age, the difference in the size of the suckling pigs with the 


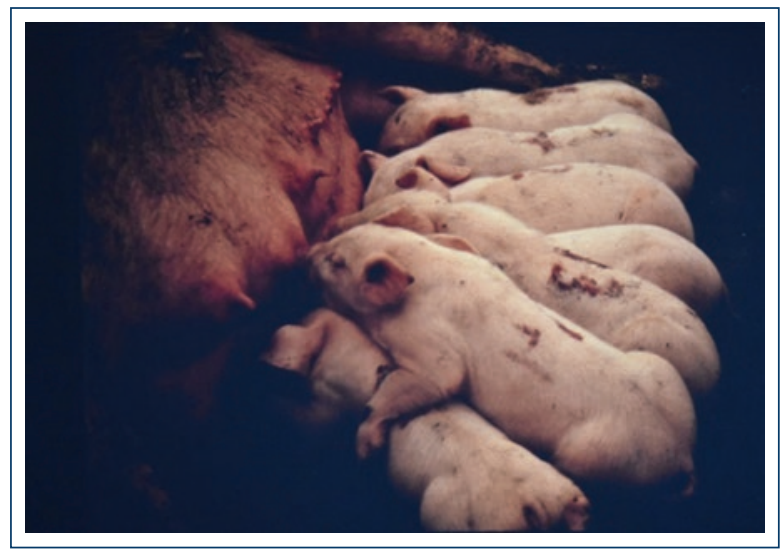

Figure 2. Photo that shows the piglets fed with the mother.

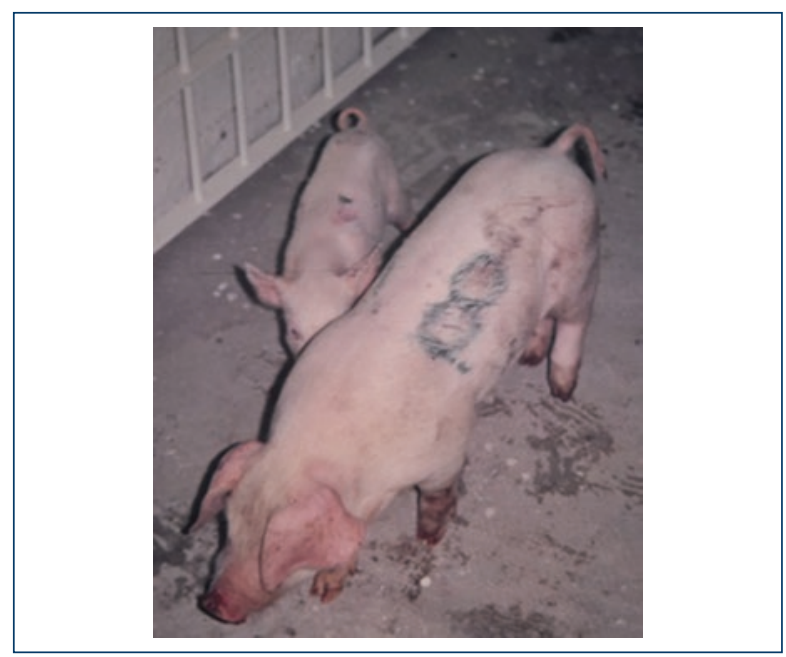

Figure 3. Photo that shows the piglets of 1 month of age: the small one fed with breast milk substitute and the other one fed with the mother.

mother in relation to the piglets suckled with BM substitute. The piglets suckled with the mother had normal size and the lactates with BM substitute gained very little weight (Fig. 3).

\section{Serotypes}

Two hundred and eighty-five isolates of $E$. coli were obtained from piglets with and without diarrhea. The largest amount of isolates obtained of piglets suckled with BM belonged to serotype 0103:H28 (62.3\%), O166:H21 (15.8\%), O98:H? (14.9\%), and O109:H40 (7.0) preferrently and the largest number of isolates obtained of piglets suckled with milk substitute belonged to
O166:H21 (59.6\%), O109:H40 (26.6\%), and O103:H28 $(13.8 \%)$ serotypes preferrently. Serotypes belonged to bloody diarrhea were 0103:H28 (1.0\%) and 0166:H21 (1.0\%) (Fig. 4).

\section{Discussion}

The present report describes what is believed to be the first study in México and probability to Mundial level. This is because, at birth, piglets are devoid of gamma globulins, because the epitelio chondrial placenta of the sows does not allow the passage of immunoglobulins to the fetus, and passive immunity can be acquired through colostral milk during the first $36 \mathrm{~h}$ of life. The lack of immunological protection causes the death of piglets ${ }^{1}$.

At present, there is a little information regarding isolates of $E$. coli of piglets suckled with BM and piglets suckled with a maternal milk substitute. In the present study, we work with two piglets groups: the first one was suckled with BM and the second was suckled with BM substitute.

The study was followed for a month, during which the body development of the group of piglets suckled with the mother was normal, unlike the group of piglets suckled with BM substitute, which showed little body development (Fig. 3). Similarly, the group breastfed with the mother only had $7(38.8 \%)$ episodes of diarrhea, as opposed to the one breastfed with milk substitute, which presented 18 (100\%) episodes of diarrhea, two with inflammatory diarrhea.

This could be because colostrum and BM are a complex physiological fluid with multifunctional roles within the gastrointestinal tract that facilitate the successful postnatal adaptation of the newborn by stimulating cellular growth and digestive maturation. Simultaneously, BM provides nutrients and bioactive factors that facilitate the adaptive functional changes required for the optimal transition from intrauterine to extrauterine life.

It is known that besides nutrients and antibodies, BM. Diaz-Jouanen and Williams ${ }^{5}$ contains cells and bioactive factors, as hormones and cytokines ${ }^{7}$ as well as growth factors such as cortisol, insulin, prolactose, tyrosine, EGF, and transforming growth factor ${ }^{19}$. These factors present in maternal milk can play a role in the regulation of the growth and differentiation of several neonatal tissues, including the immune and neuroendocrine systems. The EGF participate in the development of the intestinal mucosa, indeed, represents the site where is carried out the digestion and absorption of the nutrients ${ }^{10}$. All these factors participate 


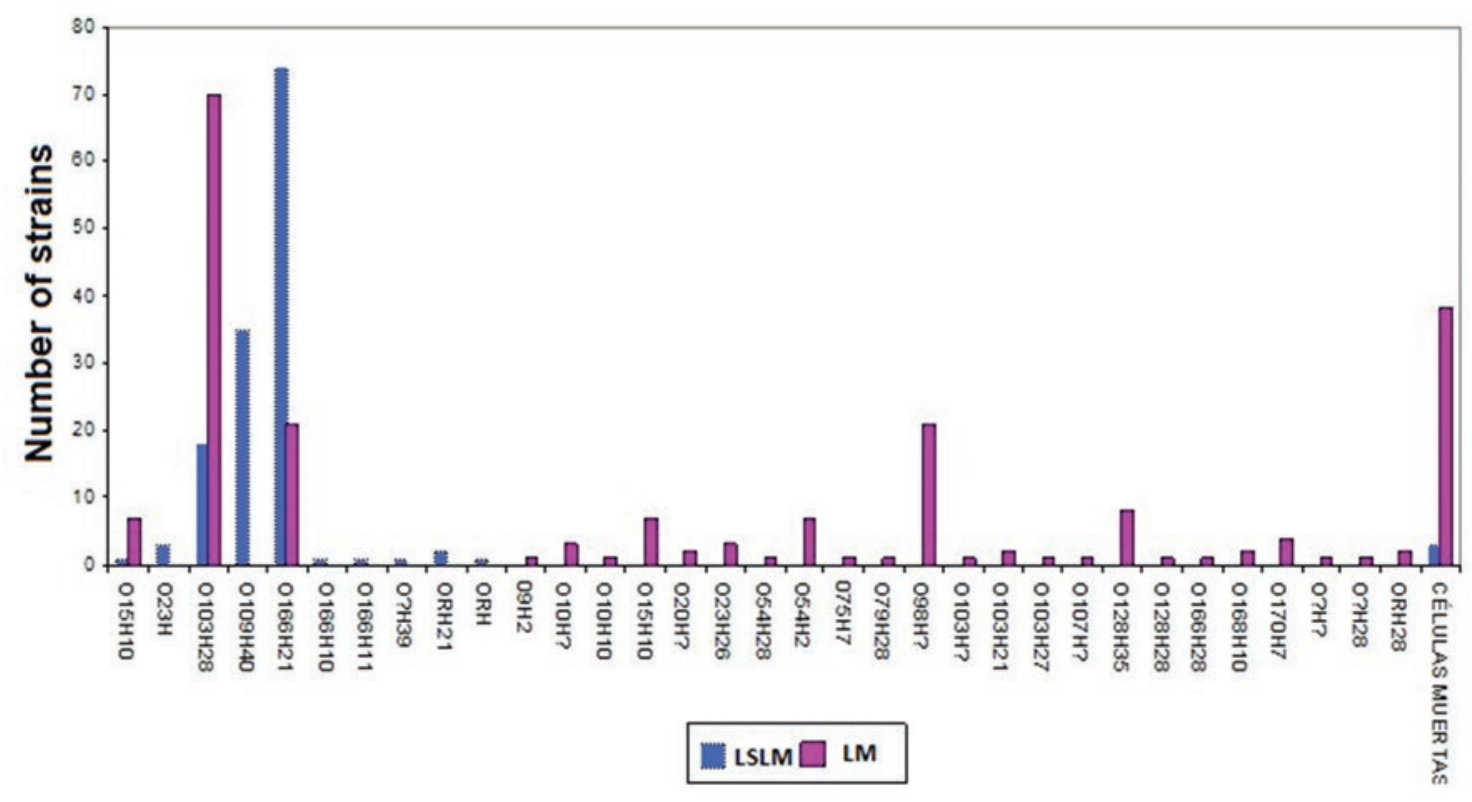

Figure 4. Serotypes of Escherichia coli isolated from piglets lactated with the mother and with breast milk substitute.

in the maturation of the immune system and in particular of the Gut-associated lymphoid tissue, and this favors the protection of the individual in the presence of pathogens.

On the other hand, the early weaning in the piglet can affect the physiological development, the corporal growth, the behaviors, cortisol levels, regulation of the hypothalamus-pituitary-adrenal axis, and modify the immune response. These changes must lead to the separation of the dam to a great extent, to coexist with piglets of other litters, as well as to the deficiency of the factors with the immunological potential present in maternal milk.

All isolates of piglets suckled with BM as the suckled with BM substitute by VITEK showed that $100 \%$ of isolated belonged to $E$. coli. It has been reported that suckling piglets eat considerable amounts of their sow's feces and $E$. coli cells are among the earliest Gram-negative bacteria to colonize the gut in pigs. Diarrhea episodes of piglets suckling with BM were on the $5^{\text {th }}$ day of births, which is able to acquire from maternal feces, skin, and teats ${ }^{1}$. Diarrhea episodes of piglets suckling with BM substitute were on the $2^{\text {nd }}$ day of births this could be doubt to the type of lactation, or infections ${ }^{3}$. Infections at this age could be due to this piglets group lack of immunological protection, because it did not breastfeeding, and we know that piglets protection is acquired from colostrum and $\mathrm{BM}^{6}$.
The predominant $E$. coli serotypes in the 285 isolates were 0103:H28 for isolates from the group breastfed with the mother and 0166:H21 from the group breastfed with BM substitute (Fig. 4). The isolates of piglets with bloody diarrhea, belonged to serotypes O103:H28 and $0166: \mathrm{H} 21$. The piglets suckled with BM, without diarrhea, the prevalent serogroup it was 0103:H28.

It is noteworthy that the serotype of E. coli O103:H28 identified both in the group of piglets suckled with BM substitute and in the group suckled with the mother, only caused inflammatory diarrhea in the piglets of the group suckled with substitute $\mathrm{BM}$, this indicates that $E$. coli from both groups of piglets belonged to the $E$. coli STEC pathotype. The fact that the genes of $E$. coli producing Shiga toxins have been expressed only in the group of suckled piglets with BM substitute, this could be due to the microenvironment of the intestine of the piglets suckled with BM substitute, which favored the expression of STx genes, giving rise to bloody diarrhea.

These serotypes could belong to non-0157 serotypes; this, taking into account that serogroup 0:103 was the first to be identified as $E$. coli no $0157^{20}$. Although serotypes differ in their virulence, the incidence and severity of infections cannot be attributed solely to the virulence factors of the pathogen are the result of the interaction of the pathogen with host and environmental factors.

E. coli producing Shiga toxin (STEC) has been associated with hemorrhagic colitis and hemolytic uremic 
syndrome in humans, however, in pigs has a high involvement in the disease of edema. Regarding the serotypes of E. coli O103: $\mathrm{H} 28,0166: \mathrm{H} 21$, and $0: 109: \mathrm{H} 40$, these are not found in the literature for E. coli, producing Shiga toxin, they could belong to the serotypes of $E$. coli No O157:H778.

The present work showed the importance of BM both in the body development of the piglet and in the protection against infections.

\section{Ethical disclosures}

Protection of human and animal subjects. The authors declare that the procedures followed were in accordance with the regulations of the relevant clinical research ethics committee and with those of the Code of Ethics of the World Medical Association (Declaration of Helsinki).

Confidentiality of data. The authors declare that they have followed the protocols of their work center on the publication of patient data.

Right to privacy and informed consent. The authors declare that no patient data appear in this article.

\section{Conflicts of interest}

The authors declare that they have no conflicts of interests.

\section{Acknowledgments}

We wish to thank Eng. David Limón Cruz for elaborating the figures. This work was supported by Facultad de Medicina, Universidad Nacional Autónoma de México.

\section{References}

1. Pérez FA. Prácticas de manejo del lechón en maternidad: estrategias para mejorar su sobrevida y aumentar la productividad. Rev Electrón Vet. 2009;11:1-21.

2. Drasar BS, Barrow PA. Intestinal Microbiology. Washington D.C: American Society for Microbiology; 1985. p. 33-5.

3. Villalpando S, Hamosh M. Early and late effects of breastfeeding: does breast-feeding really matter? Biol Neonate. 1998;74:107.

4. Bertschinger HU. Postweaning Escherichia coli diarrhea and edema disease. In: Straw BE, DAllaire S, Mengeling WL, Taylor DJ, editors. Disease of Swine. $8^{\text {th }}$ ed. Ames: lowa State University Press; 1999. p. 441-54.

5. Diaz-Jouanen E, Williams RC Jr. T and B lymphocytes in human colostrum. Clin Immunol Immunopathol. 1974;3:248-55.

6. Hanson LA, Winberg J. Breast milk and defence against infection in the newborn. Arch Dis Child. 1972;47:845-8.

7. Andreas NJ, Hyde MJ, Herbert BR, Jeffries S, Santhakumaran S, Mandalia S, et al. Impact of maternal BMI and sampling strategy on the concentration of leptin, insulin, ghrelin and resistin in breast milk across a single feed: a longitudinal cohort study. BMJ Open. 2016;6:e010778.

8. Wagner CL, Taylor SN, Johnson D. Host factors in amniotic fluid and breast milk that contribute to gut maturation. Clin Rev Allergy Immunol. 2008;34:191-204.

9. Julius $\mathrm{MH}$, Janusz $\mathrm{M}$, Lisowski J. A colostral protein that induces the growth and differentiation of resting B lymphocytes. J Immunol. 1988;140:1366-71.

10. Warner BW, Warner BB. Role of epidermal growth factor in the pathogenesis of neonatal necrotizing enterocolitis. Semin Pediatr Surg. 2005; $14: 175-80$

11. Hill DR, Newburg DS. Clinical applications of bioactive milk components. Nutr Rev. 2015;73:463-76.

12. Rohde T, MacLean DA, Klarlund Pedersen B. Glutamine, lymphocyte proliferation and cytokine production. Scand J Immunol. 1996;44:648-50.

13. Castro AM. In Bacteriología Médica Basada en Problemas. $2^{\text {nd }}$ ed. México: Manual Moderno; 2014. p. 115-43.

14. Mainil JG, Daube G. Verotoxigenic Escherichia coli from animals, humans and foods: who's who? J Appl Microbiol. 2005;98:1332-44.

15. Dubreuil JD, Isaacson RE, Schifferli DM. Animal enterotoxigenic Escherichia coli. EcoSal Plus. 2016;7:1-79.

16. Nagy B, Fekete PZ. Enterotoxigenic Escherichia coli (ETEC) in farm animals. Vet Res. 1999;30:259-84.

17. Garabal JI, González EA, Vázquez F, Blanco J, Blanco M, Blanco JE, et al. Serogroups of Escherichia coli isolated from piglets in Spain. Vet Microbiol. 1996;48:113-23.

18. Luna-Gierke RE, Griffin PM, Gould LH, Herman K, Bopp CA, Strockbine $\mathrm{N}$, et al. Outbreaks of non-O157 shiga toxin-producing Escherichia coli infection: USA. Epidemiol Infect. 2014;142:2270-80.

19. Castro AM, Porras-Villalobos MG. La protección de la leche maternal a los recién nacidos. Una vision actualizada. Rev Mex Pediatr. 2003;70:23-6.

20. Kalchayanand N, Terrance MA, Bosilevac JM, Wheeler TM. Non-0157 Shiga Toxin-producing Escherichia coli: prevalence Associated with Meat Animals and Controlling Interventions. Proceedings of American Meat Science Association $64^{\text {th }}$ Annual Reciprocal Meat Conference. Manhattan Kansas: Kansas State University; 2012. p. 1-9. 